FERMILAB-Conf-97/215-E

CDF

\title{
Properties of Events with a Rapidity Gap Between Jets in CDF
}

\author{
Philip Mélèse \\ For the CDF Collaboration \\ The Rockefeller University \\ New York, New York
}

Fermi National Accelerator Laboratory

P.O. Box 500, Batavia, Illinois 60510

June 1997

Presented at the 5th International Workshop on Deep Inelastic Scattering and QCD (DIS'97), Chicago, Illinois, April 14-18, 1997 


\section{Disclaimer}

This report was prepared as an account of work sponsored by an agency of the United States Government. Neither the United States Government nor any agency thereof, nor any of their employees, makes any warranty, expressed or implied, or assumes any legal liability or responsibility for the accuracy, completeness, or usefulness of any information, apparatus, product, or process disclosed, or represents that its use would not infringe privately owned rights. Reference herein to any specific commercial product, process, or service by trade name, trademark, manufacturer, or otherwise, does not necessarily constitute or imply its endorsement, recommendation, or favoring by the United States Government or any agency thereof. The views and opinions of authors expressed herein do not necessarily state or reflect those of the United States Government or any agency thereof.

\section{Distribution}

Approved for public release; further dissemination unlimited. 
Rockefeller University Report: RU 97/E-41

CDF note: CDF/4223

\title{
Properties of Events with a Rapidity Gap Between Jets in CDF ${ }^{*}$
}

\author{
Philip Mélèse \\ The Rockefeller University, New York \\ (The CDF Collaboration)
}

\begin{abstract}
We present the $\eta$ and $E_{T}$ dependence of dijet events produced by color-singlet exchange in $\bar{p} p$ collisions at $\sqrt{s}=1.8 \mathrm{TeV}$ using data collected by the CDF collaboration at the Fermilab Tevatron. In a sample of events with two forward jets, where the jets are on opposite sides in rapidity with $|\eta|>1.8$ and $E_{T}>20 \mathrm{GeV}$, we find $1.13 \pm 0.12$ (stat) \pm 0.11 (syst)\% have a rapidity gap between the jets consistent with color-singlet exchange. This signal has no significant dependence on the jet $E_{T}$ or the rapidity interval between the jets.
\end{abstract}

\section{INTRODUCTION}

In high energy $\bar{p} p$ collisions, jets are normally produced through the exchange of a quark or gluon between partons in the interacting hadrons. Because of the exchanged color, there are typically particles produced in the rapidity [1] region between the jets. The signature of dijets produced by color-singlet exchange is the absence of particles between the jets. In a simple two-gluon color-singlet model, the fraction of dijet events with a rapidity gap, compared to all dijet events, was estimated to be $\sim 10^{-2}[2,3]$, while the fraction of gap events due to electroweak exchange is only $\sim 10^{-4}[3]$ for $\bar{p} p$ production at $\sqrt{s}=1.8 \mathrm{TeV}$.

The fraction of jets produced by color-singlet exchange has been previously measured at $\sqrt{s}=1.8 \mathrm{TeV}$ by both the CDF and D0 collaborations at the Fermilab Tevatron, and in photoproduction by the ZEUS Collaboration [6] at HERA. CDF [4] has measured $\left[0.85 \pm 0.12(\text { stat })_{-0.12}^{+0.24}(\right.$ syst $\left.)\right] \%$ for dijets with leading (most energetic) jet $E_{T}>40 \mathrm{GeV}$; D0 [5] measured [1.07 \pm

*) Presented at the Fifth International Workshop on Deep Inelastic Scattering and QCD, DIS97, Chicago, U.S.A, 14-18 April 1997. 
$0.10(\text { stat })_{-0.13}^{+0.25}($ syst $\left.)\right] \%$ for $E_{T}^{\text {jet }}>30 \mathrm{GeV},|\eta|>2$ and $\eta_{1} \eta_{2}<0$; and ZEUS [6] measured $\approx 7 \%$ at $\sqrt{s} \sim 150 \mathrm{GeV}$ for jets of $E_{T}>6 \mathrm{GeV}$. The magnitude of the signal in $\bar{p} p$ collisions is consistent with the strongly interacting colorsinglet prediction, and much larger than the electroweak prediction. In all cases, the background to the rapidity gap signal was determined using a fit to a multiplicity distribution between the jets and extrapolating to the lowmultiplicity (gap) bins. The color-singlet signal is defined as the excess over this background.

The result presented here uses a background (non-singlet) multiplicity template from the data, which eliminates the systematic uncertainty in the previous results due to extrapolating the multiplicity function to the lowmultiplicity bins. We also present the dependence of the color-singlet signal on the $E_{T}^{j e t}$ and the rapidity interval between the jets, $\Delta \eta^{j e t}$.

\section{DETECTOR AND DATA SAMPLE}

The CDF detector is described in detail elsewhere [7]. The detector components relevant to this study are the Central Tracking Chamber (CTC), which detects charged particles, and the calorimeters, which detect both charged and neutral particles. The CTC tracking efficiency varies from $\sim 60 \%$ for $P_{T}=300$ $\mathrm{MeV}$ to over $95 \%$ for $P_{T}>400 \mathrm{MeV}$ within $|\eta|<1.2$, and falls monotonically beyond $|\eta|=1.2$ to zero at $|\eta| \sim 1.8$. For this analysis, a "charged particle" is a reconstructed 3 -dimensional track with $P_{T}>300 \mathrm{MeV}$. The central calorimeter covers the regions $|\eta|<1.1$ with projective towers of dimension $\Delta \eta=0.1 \times \Delta \phi=15^{\circ}$. The "tower multiplicity" is defined as the number of towers with a measured $E_{T}>200 \mathrm{MeV}$, which corresponds approximately to a true $E_{T}>300 \mathrm{MeV}$.

The data comes from a forward dijet trigger with offline requirements of two jets with $E_{T}^{\text {jet }}>20 \mathrm{GeV}$ and $1.8<|\eta|<3.5$, where the jets are on opposite sides in $\eta$ (i.e. $\eta_{1} \eta_{2}<0$ ). In order to avoid multiple interaction events, which tend to wash out any rapidity gap, we require that there be only one reconstructed vertex in the event. The remaining sample consists of 10,200 opposite-side dijet events. Events with the same selection, except that both jets are on the same side $\left(\eta_{1} \eta_{2}>0\right)$ were used to measure the rate of diffractive dijets [8], and provide a control sample in this analysis for the multiplicity in the central region with no color-singlet contribution.

\section{RESULTS}

The color-singlet signature is an excess of low track or tower multiplicity between the jets in the opposite-side dijet sample compared to the sameside control sample. Figure 1a (b) shows the track (tower) multiplicities for opposite-side and same-side dijet events. The bin-by-bin asymmetry in Fig. 1c 
shows the excess in the zero track bin, which is consistent with the signal in the lowest 3 bins of the tower multiplicity (Fig. 1d). The fractional excess of rapidity gaps between jets is corrected for the vertex selection efficiency to determine the fraction of dijet events produced by color singlet exchange: $R=[1.13 \pm 0.12($ stat $) \pm 0.11$ (syst) $] \%$ (preliminary)

for jet $E_{T}>20 \mathrm{GeV}$ and $\left|\eta_{1}, \eta_{2}\right|>1.8$. This value is consistent with the previously published results $[4,5]$.

By selecting opposite-side dijet events with zero tracks and less than 3 towers in the central region between the jets, we can separate out 221 color-singlet candidates which contain only $\sim 25 \%$ non-singlet background events. This sample is used to study the $E_{T}^{j e t}$ and $\Delta \eta^{j e t}$ dependence of the color-singlet produced dijets as a fraction of all dijets. Because the color-singlet sample contains some non-singlet background and the requirement of low multiplicity between the jets may bias the jet distributions, we compare the color-singlet sample with a low-multiplicity "control" sample defined as events with 1, 2 or 3 tracks and less than 7 towers in the central region between the jets.

Figures $2 \mathrm{a}, \mathrm{b}$ and $\mathrm{c}$ show the mean dijet $E_{T}$, the third jet $E_{T}$ and the dijet $\eta$ separation $\left(\left|\eta_{1}-\eta_{2}\right| / 2\right)$ dependence of the ratio of color-singlet to all dijets, normalized to unity. There is no dependence on mean dijet $E_{T}$ of the color-singlet signal compared to the entire sample, or the low-multiplicity control sample. Because both the color-singlet and low-multiplicity samples restrict the activity in the central region between the two leading jets, the ratio to all events decreases with third jet $E_{T}$ (Fig. $2 \mathrm{~b}$ ). The color-singlet signal dependence on the $\eta$ separation, Fig. $2 c$, is statistically consistent with the entire sample, although there is some hint that the color-singlet events are reduced at higher $\eta$ separation. The color-singlet fraction is fairly independent on jet $E_{T}$ and $\Delta \eta$, which is consistent with the two-gluon model of Ref. [2].

\section{REFERENCES}

1. The pseudorapidity is defined: $\eta=-\ln \tan \frac{\theta}{2}$, where $\theta$ is the polar angle with respect to the proton beam. We use $\eta$ and rapidity interchangeably.

2. J.D. Bjorken, Phys. Rev. D47, 101 (1993).

3. H. Chehime et al., Phys. Lett. B286, 397 (1992).

4. F. Abe et al. ( CDF), Phys. Rev. Lett. 74, 855 (1995).

5. S. Abachi et al. (D $\varnothing)$, Phys. Rev. Lett. 76, 734 (1996).

6. M. Derrick et al. (ZEUS), Phys. Lett. B369, 55 (1996).

7. F. Abe et al., Nucl. Instrum. Methods A271, 387 (1988).

8. F. Abe et al., "Measurement of Diffractive Dijet Production at the Fermilab Tevatron", submitted to Phys. Rev. Letters. 

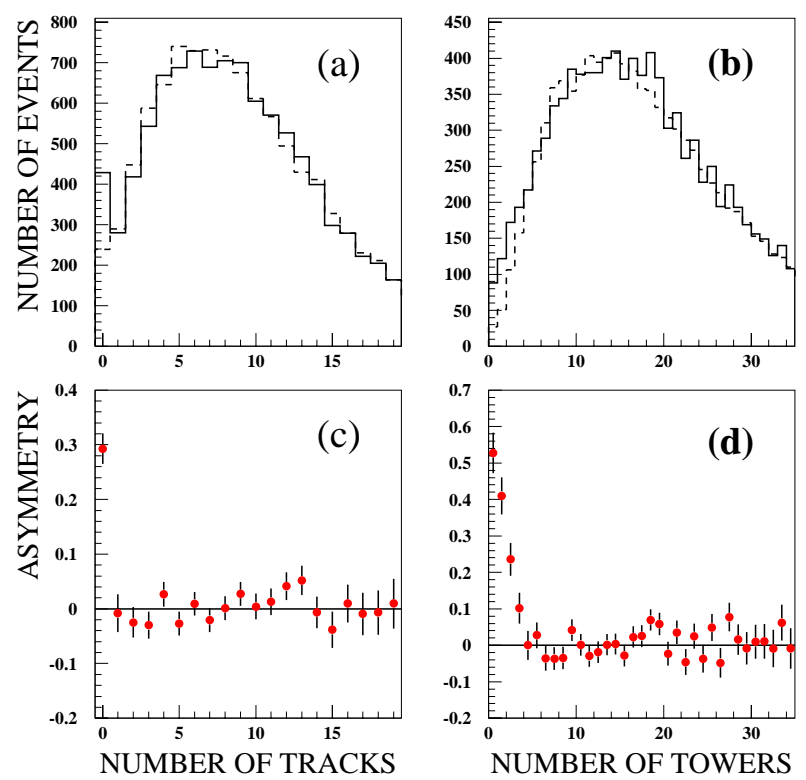

FIGURE 1. The multiplicity distributions (a) for tracks and (b) for calorimeter towers in the regions $|\eta|<1.0$ for opposite-side dijet events (solid), and $|\eta|<1.2(|\eta|<1.25)$ for tracks (towers) of same-side dijet events (dashed). The bin-by-bin asymmetry for tracks (c) and towers (d), defined as the ratio of the difference over the sum of the opposite-side and same-side multiplicity distributions.
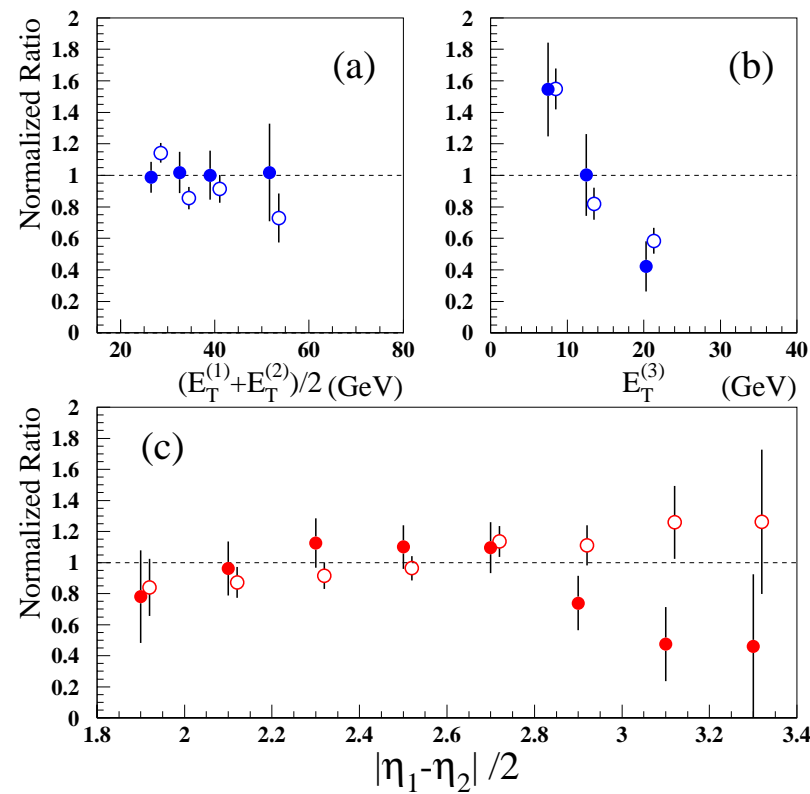

FIGURE 2. Normalized (to be unity on average) ratios of gap (solid points) over all events versus: (a) the dijet mean $E_{T}$, (b) the third jet $E_{T}$, and (c) half the $\eta$ separation between the two leading jets. The low-multiplicity control sample (open circles) is also shown for comparison. 